\title{
Exploring spin-orbital models with dipolar fermions in zig-zag optical lattices
}

\author{
G. Sun, ${ }^{1}$ G. Jackeli, ${ }^{2, \text { 困 L. Santos },{ }^{1} \text { and T. Vekua }}{ }^{1}$ \\ ${ }^{1}$ Institut für Theoretische Physik, Leibniz Universität Hannover, 30167 Hannover, Germany \\ ${ }^{2}$ Max-Planck-Institut für Festkörperforschung, Heisenbergstrasse 1, D-70569 Stuttgart, Germany
}

\begin{abstract}
Ultra-cold dipolar spinor fermions in zig-zag type optical lattices can mimic spin-orbital models relevant in solid-state systems, as transition-metal oxides with partially filled $d$-levels, with the interesting advantage of reviving the quantum nature of orbital fluctuations. We discuss two different physical systems in which these models may be simulated, showing that the interplay between lattice geometry and spin-orbital quantum dynamics produces a wealth of novel quantum phases.
\end{abstract}

Orbital degrees of freedom of electrons play an important role in the formation of various novel phases observed in transition-metal oxides with partially filled $d$-levels [1, 2]. In Mott insulators, they may enhance thermal as well as quantum fluctuations [3] and lead to spin/orbital liquid states [4 7], and to spontaneously dimerized states without any long-range magnetic order [8, 9]. In many solid-state systems, the orbital dynamics is often quenched, due to the coupling of the orbitals to Jahn-Teller phonons, and the study of the quantum nature of orbitals demands systems with strong super-exchange coupling between spins and orbitals. However, in real materials, the coupling strengths are fixed by nature, being very difficult to modify, thus limiting the experimental access to a potentially vast phase diagram.

Ultra-cold spinor gases in optical lattices open new fascinating perspectives for the analysis of the quantum nature of orbitals [10 12]. The coupling constants in these systems can be easily controlled by modifying the lattice parameters and/or by means of Feshbach resonances [13]. Different lattice geometries, including frustrated lattices, like triangular [14] and Kagomé lattices [15], may be created by combining different counter-propagating laser beams, and superlattice techniques. In addition, not only the physics in the lowest band but also that in higher bands may be controllably studied [16]. Moreover, recent experiments on Chromium [17] and Dysprosium [18] atomic gases, and polar molecules [19], are unveiling the exciting physics of ultra-cold dipolar gases, for which the dipole-dipole interactions may lead to exotic phases [20, 21].

In this Letter, we show that dipolar spinor Fermi gases in appropriate zig-zag lattice geometries allow for the quantum simulation of spin-orbital models for a family of Mott insulating compounds, including systems with weak [8, 22, 23] as well as pronounced 24] relativistic spin-orbit couplings. Moreover, these models, which are relevant for real materials, as pyroxene titanium and layered vanadium oxides, may be explored with dipolar Fermi gases in parameter regimes which are hardly accessible for solid-state compounds, allowing for the observation of novel quantum phases.

Physical realizations and effective Hamiltonian.- We
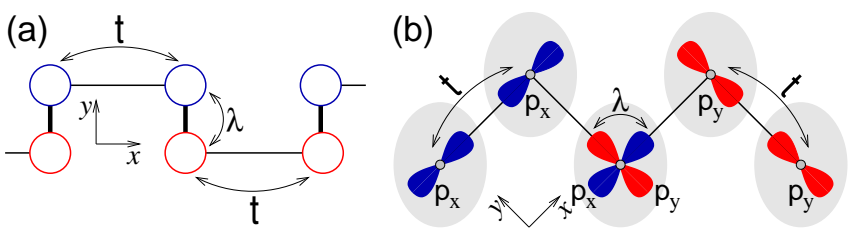

FIG. 1: (Color online) Two different systems discussed in this work. (a) Snake-like ladder with rungs (bonds) along $y(x)$, and one fermion per rung. The inter (intra)-rung hopping is indicated by $t(\lambda)$; (b) Zig-zag lattice with one fermion per site occupying either $p_{x}$ or $p_{y}$ orbital states. Along a bond in $x(y)$ direction only $p_{x}\left(p_{y}\right)$ orbitals are connected by a finite hopping amplitude $t$. A deformation of the lattice wells (shaded area) leads to a mixing between $p_{x}$ and $p_{y}$ orbitals with amplitude $\lambda$.

start by presenting two possible scenarios, $A$ and $B$, in which dipolar Fermi gases may allow for the simulation of the above-mentioned spin-orbital models.

A: First scenario is provided by a snake-like ladder as that shown in Fig. 1(a), formed by bonds and rungs along $x$ and $y$ directions, respectively. This lattice geometry may be achieved by using a combined blue-detuned ladder-like lattice and a properly aligned red-detuned zig-zag lattice [25]. The overimposed red-detuned lattice allows for enhancing the potential barrier at alternated bonds in the upper and lower legs. A sufficiently strong red-detuned lattice leads hence to broken bonds as depicted in Fig. 1(a). A similar technique has been recently employed for the realization of a Kagomé lattice [15]. The hopping within the same rung is denoted by $\lambda$, whereas the inter-rung hopping for unblocked bonds is denoted as $t$ [Fig. 1(a)]. The on-site repulsion $U$ results from the combination of dipolar and contact interactions. The dipoles are oriented in the $x z$-plane in such a way that fermions at the same rung experience maximal nonlocal repulsion $V$ and dipoles on neighboring rungs interact identically, irrespective whether they occupy upper or lower sites, with the interaction strength much weaker than intra-rung repulsion. As a result, inter-rung dipolar interaction plays no role in the discussion below (interaction between next-nearest rungs is considered negligibly small). We assume $U, V \gg t, \lambda$, and consider the case of one fermion per rung. The system is then in the 
Mott-insulator regime with one fermion localized on each rung. The sites belonging to the $i$-th rung of the ladder are distinguished by the pseudo-orbital quantum number $\sigma_{i}^{z}$, with the convention that $\sigma_{i}^{z}=+(-) 1$ when the upper (lower) site on a given rung is occupied. Defining $\alpha \equiv U / V$, and setting $t^{2} / 2 U$ as the energy unit we arrive to the effective spin-orbital Hamiltonian of KugelKhomskii type [26, 27],

$$
\begin{aligned}
H & =\sum_{i}^{N}\left[2 \mathbf{S}_{i} \mathbf{S}_{i+1}+\alpha-\frac{1}{2}\right]\left[1+(-1)^{i} \sigma_{i}^{z}\right]\left[1+(-1)^{i} \sigma_{i+1}^{z}\right] \\
& -\Delta \sum_{i}^{N} 2 \mathbf{S}_{i} \mathbf{S}_{i+1}\left[1-\sigma_{i}^{z} \sigma_{i+1}^{z}\right]-\lambda \sum_{i}^{N} \sigma_{i}^{x}
\end{aligned}
$$

where $\mathbf{S}_{i}$ is the spin- $\frac{1}{2}$ operator (stemming from the spinor nature of the Fermi gas), and $\sigma_{i}^{z, x}$ are Pauli matrices describing the pseudo-orbital variables. We have added an additional term proportional to the coupling constant $\Delta$. Although $\Delta=0$ for realization $\mathrm{A}$, it plays an important role in the alternative case $\mathrm{B}$ discussed below. Note that the ratio $\alpha$ may be modified basically at will, since $U$ and $V$ may be independently controlled using Feshbach resonances [13], altering the lattice spacings, or modifying the transversal confinement [20].

B: Second possible scenario is provided by a zigzag lattice in the $x y$-plane, loaded with spinor dipolar fermions in $p$-bands [16] [Fig. [1(b)], where the dipoles are oriented along $z$-axis. Assuming a strong confinement along $z$, we retain two degenerate orthogonal $p_{x}$ and $p_{y}$ orbitals per lattice site. In this realization, $t$ denotes the hopping between similar orbitals at neighboring wells [Fig. 1(b)]. An in-plane deformation of the lattice wells (e.g. by an additional weak tilted lattice) leads to a mixing of the $p_{x}$ and $p_{y}$ orbitals within the same well with an amplitude $\lambda$.

The interaction parameters for two fermions within the same well are on-site repulsions $U$ (within the same orbitals) and $V$ (among different orbitals), and Hund's exchange $J_{H}$ [27]. Two fermions occupying the same orbital may form a symmetric or an antisymmetric state with respect to the orbital index with corresponding energies $U+J_{H}$ and $U-J_{H}$, which are split by the so-called pair-hopping term with an amplitude $J_{H}$ 27]. The overall energy scale will now be modified to $t^{2} / 2 \tilde{U}$, where $\tilde{U}=\left(U^{2}-J_{H}^{2}\right) / U$. When two fermions occupy different orbitals, they may form a spin-singlet or a spintriplet state with corresponding energies $V+J_{H}$ and $V-J_{H}$, which are split by Hund's exchange. In the strong coupling limit $U \pm J_{H}, V \pm J_{H} \gg t, \lambda$ and with one particle per lattice well the system is in the Mottinsulator regime, and we arrive at Hamiltonian (1) with $\Delta=J_{H} \tilde{U} /\left(V^{2}-J_{H}^{2}\right)$ [27]. Now, $\alpha$ also gets modified accordingly, $\alpha=\tilde{U}\left(V+J_{H} / 2\right) /\left(V^{2}-J_{H}^{2}\right)$.

Note that for a purely contact interaction $V=J_{H}$ and in the triplet channel two fermions do not experience any repulsion [27]. Thus, without dipolar couplings, the Mott phase of one fermion per well would not be stable. In the Mott-insulator regime, one can still vary $\Delta$ in a wide range by changing the relative ratio of the strengths of the contact and dipolar interactions.

The model (11), at $\alpha \simeq 1$, describes the spin-orbital interplay in Mott insulating transition-metal compounds. At $\lambda=0$, it describes a zig-zag chain of spin one-half $\mathrm{Ti}^{3+}$ ions, with active $d_{x y}$ and $d_{y z}$ orbitals, in pyroxene titanium oxides $A \operatorname{TiSi}_{2} \mathrm{O}_{6}(A=\mathrm{Na}, \mathrm{Li})[22,23]$. On the other hand, for $\Delta=0$ the Hamiltonian (11) represents the 1D counterpart of the 2D model for $\mathrm{Sr}_{2} \mathrm{VO}_{4}$ [24]. In the latter, the role of spins in Eq. (1) is played by an isospin variable discerning the Kramers partners, while the pseudo-orbital variables distinguishes two lowest Kramers doublets of $\mathrm{V}^{4+}$ ion, and $\lambda$ term in Eq. (1) represents relativistic spin-orbit coupling.

In the following we study the ground-state properties of the model (11) in the wide parameter range by analytical and complementary numerical approaches.

Ground-state phase diagram for $\Delta=0$.- We first discuss the case $\Delta=0$ relevant for realization A. At $\lambda=0$ the orbitals become classical and the ground-state phase diagram is easily mapped. For $\alpha>2$, the groundstate is $2 \times 2^{N}$ degenerate: there is anti-ferro (AF) order in orbitals $\left\langle\sigma_{i}^{z}\right\rangle= \pm(-1)^{i}$ ( \pm refers to two degenerate AF states), whereas the spin part is completely degenerate (bold line on Fig. 2(a)). For $\alpha>2$ and $\lambda \rightarrow 0$, the system is described by an effective spin model, $H_{S} \sim \lambda^{2} \sum_{i} \mathbf{S}_{i} \mathbf{S}_{i+1}$, an isotropic Heisenberg antiferromagnet (iH). Thus, quantum fluctuations of orbitals, induced by $\lambda$-term, lifts immediately the infinite degeneracy of the ground state, resembling order from disorder. We denote this phase (iH,AF), where the first term denotes $\mathrm{iH}$ spin phase, and the second term $\mathrm{AF}$ orbital phase. We employ a similar notation from now on. Higher order terms in $\lambda / \alpha$ cannot break the $S U(2)$ spin and translational symmetries, and thus for $\lambda \rightarrow 0$ the $\mathrm{iH}$ phase in spin degrees is stable. As shown below, the $\mathrm{iH}$ is recovered for strong $\lambda$ independently of the value of $\alpha$. Thus, we can expect that for $\alpha>2$ there is an unique iH phase in spin degrees of freedom for any $\lambda \neq 0$. On the contrary, with increasing $\lambda$ the orbital degrees of freedom experience an Ising transition from AF to paramagnetic $(\mathrm{P})$ phase with $\left\langle\sigma_{i}^{z}\right\rangle=0$.

For $\lambda=0$ and $0<\alpha<2$, the exact ground state is two-fold degenerate and represents a direct product of ferro (F) orbital order, $\left\langle\sigma_{i}^{z}\right\rangle=+1(-1)$, and spontaneously dimerized Majumdar-Ghosh (MG) state [28] in spins, with spin-singlets located on odd (even) bonds. We call this phase a dimer-ferro $(\mathrm{D}, \mathrm{F})$. An infinitesimal $\lambda$ generates an exchange between the disconnected nearestneighbour dimers, $\sim \lambda^{4} \sum \mathbf{S}_{2 i+1} \mathbf{S}_{2 i+2}$. With increasing $\lambda$, the dimerization order in spins, $D=\frac{1}{N} \sum_{i} \mid\left\langle\mathbf{S}_{i} \mathbf{S}_{i+1}-\right.$ $\left.\mathbf{S}_{i} \mathbf{S}_{i-1}\right\rangle \mid$, disappears together with the orbital ferro order at a Kosterlitz-Thouless (KT) phase transition [29]. 

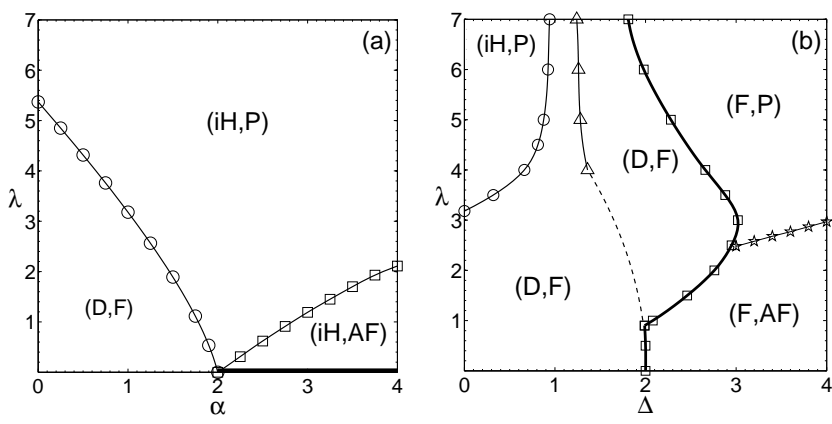

FIG. 2: Ground state phase diagram of the model (1) for (a) $\Delta=0$ and (b) $\alpha=1$. First and second phases in parentheses refer to spin and orbital sectors, respectively. See text, for a description of phases and phase transitions.

A numerical ground-state phase diagram of the model (1) for $\Delta=0$ is presented in Fig. 2(a) (details of the numerical simulations are discussed later).

Ground-state phase diagram for $\Delta \neq 0$.- We now turn to the case of finite $\Delta$. We focus on the regime $\alpha=1$ relevant to realistic condensed-matter systems $8,22,24]$. For $\lambda=0$, a simple calculation shows that the ground state is $(\mathrm{F}, \mathrm{AF})$ for $\Delta>2(2-\alpha)$ and $(\mathrm{D}, \mathrm{F})$ for $\Delta<2(2-\alpha)$. Note, that once the spin dimerization pattern is spontaneously chosen, the direction of orbitals becomes unambiguously selected, thus Ising $Z_{2}$ orbital order is 'slaved' by translation symmetry breaking. Whereas for $\Delta=0$ an infinitesimal $\lambda$ induces AF spin-exchange $\propto \lambda^{4}$ on interdimer (weaker) bonds in the $(\mathrm{D}, \mathrm{F})$ phase, for finite $\Delta$ the leading spin-exchange along the weaker bonds is instead ferromagnetic $\sim-\lambda^{2} \Delta \sum \mathbf{S}_{2 i+1} \mathbf{S}_{2 i+2}$. Hence there is a competition between the $\Delta$ and $\lambda$ terms promoting, respectively, a $\mathrm{F}$ and $\mathrm{AF}$ character of the weaker bonds. The character of spin correlations on weak bonds $\left\langle\mathbf{S}_{2 i+1} \mathbf{S}_{2 i+2}\right\rangle$ changes from AF (small $\Delta$ region) to $\mathrm{F}$ (larger $\Delta$ region) across $\Delta_{M G}=\lambda^{2} / 4+O\left(\lambda^{4}\right)$ line, resembling the behaviour across the MG point in the spin- $\frac{1}{2}$ $j_{1}-j_{2}$ model [30].

For $\lambda \rightarrow \infty$ the orbital degrees of freedom are quenched. The system becomes equivalent to a $S U(2)-$ symmetric spin- $\frac{1}{2}$ chain, which up to $\mathcal{O}\left(\lambda^{-2}\right)$ is described by the effective Hamiltonian,

$$
H_{S}=\sum_{i, n}\left[j_{n} \mathbf{S}_{i} \mathbf{S}_{i+n}+\Omega\left(\mathbf{S}_{i-1} \mathbf{S}_{i}\right)\left(\mathbf{S}_{i+1} \mathbf{S}_{i+2}\right)\right]
$$

where $j_{1}=2-2 \Delta+O\left(\lambda^{-1}\right), j_{2}=\lambda^{-1}, j_{3}, \Omega$ are both of order $\lambda^{-2}$, and the longer range exchanges are suppressed as $j_{n} \sim O\left(\lambda^{1-n}\right)$ 27]. There are two clear phases in this regime, (iH,P) for $1-\Delta \gg \lambda^{-1}$ and $(\mathrm{F}, \mathrm{P})$ for $1-\Delta \ll$ $-\lambda^{-1}$.

One may suspect for large $\lambda$ a direct $(\mathrm{iH}, \mathrm{P})$ to $(\mathrm{F}, \mathrm{P})$ transition with growing $\Delta$. This, however, is not the case, as can be shown by a bosonization analysis of the effective spin model (2). Starting from (iH,P) state, with increasing $\Delta$ the system necessarily enters first into a dimerized state via a $\mathrm{KT}$ phase transition at $\Delta_{K T} \simeq$ $1-1 / 2 \lambda$. At larger $\Delta$, a MG state will be approximated at $\Delta_{M G} \simeq 1+1 / 2 \lambda$, where dimerization will reach the value $D \simeq 3 / 4$. Increasing further $\Delta$, at $\Delta^{\prime} \simeq 1+3 / 2 \lambda$, nearest-neighbor coupling in Eq. (2) vanishes, $j_{1}\left(\Delta^{\prime}\right)=0$, and the $1 / \lambda^{2}$ terms, $j_{3}$ and $\Omega$, are the leading ones that couple two sub-chains 27]. Bosonization shows [27] that, inspite of these terms, in the large $\lambda$ limit, the system behaves at low energies as two decoupled spin- $\frac{1}{2}$ chains, albeit at $\Delta^{*}=\Delta^{\prime}+O\left(\lambda^{-2}\right)$. Hence around the $\Delta=\Delta^{*}$ line the effective spin model in strong coupling is described by a $j_{1}-j_{2}$ model, where $j_{1}$ changes sign from antiferro (for $\Delta<\Delta^{*}$ ) to ferro (for $\Delta>\Delta^{*}$ ), whereas $j_{2}$ stays positive. Bosonization, supported by recent numerical studies, predicts that the ground state of two weakly coupled spin- $\frac{1}{2}$ chains is dimerized irrespective of the sign of $j_{1}$ coupling 31 33]. Hence, in our case there is a special fine-tuning line bisecting the dimerized phase, $\Delta=\Delta^{*}$ line, described by double KT phase transition [34] where spin dimerization and ferro orbital order both vanish. Finally, there is a first order phase transition line separating $(\mathrm{D}, \mathrm{F})$ and $(\mathrm{F}, \mathrm{P})$ states at $\Delta_{F} \simeq 1+7 / 2 \lambda$.

This sequence of phases and phase transition curves $\left(\Delta_{K T}, \Delta^{*}, \Delta_{F}\right)$, which has been established analytically for large $\lambda$ with the help of effective spin model (2), has been confirmed for $\lambda \gtrsim 4$ by numerical simulations (discussed below) of the original model (11).

Numerical procedures.- The phase diagrams depicted in Figs. 2(a,b) were obtained by means of a combination of Lanczos exact diagonalization, density matrix renormalization group simulations based on matrix product states (MPS) [35] and the infinite time-evolving block decimation (iTEBD) algorithm [36], confirming the analytical predictions for $\lambda \rightarrow 0$ and $\lambda \rightarrow \infty$.

The KT transition between $(\mathrm{D}, \mathrm{F})$ and $(\mathrm{iH}, \mathrm{P})$ was extracted by Lanczos method from the extrapolation of the level crossing 37. between the first excited singlet of the $(\mathrm{D}, \mathrm{F})$ phase and the first excited triplet of the $(\mathrm{iH}, \mathrm{P})$ phase for systems of up to $N=12$ rungs/wells. Ising transition lines were obtained, by MPS simulations, from the peak in the fidelity susceptibility [38], and that on Fig. 2(b) between (F,AF) and (F,P) accurately follows the analytical line $\lambda=\Delta / 2+\alpha$. The first order phase transition into the $(\mathrm{F}, \mathrm{AF})$ or $(\mathrm{F}, \mathrm{P})$ states [bold line in Fig. 2(b)] was obtained by MPS method, from the jump of the ground-state total spin from 0 (singlet state) to a fully polarized $N / 2$ (ferro state) [39]. In MPS simulations we have used periodic boundary conditions and system sizes of up to $N=32$ rungs/wells were considered. For $\lambda \gtrsim 4$, the double KT phase transition line bisecting the (D,F) phase [see Fig. 2(b)] was determined from vanishing dimer order using iTEBD method. For smaller values of $\lambda(1<\lambda \lesssim 4)$, around the dashed line of Fig. 2 (b), our simulations indicate an intermediate quadrumerized phase [40]. 
Final remarks.- We have assumed above a unit occupation per rung/well, for which dipolar interactions were necessary for realizing Mott insulating state. The case of two fermions per rung/well does not require dipolar interactions. In the case of the snake-like lattice [see Fig.11(a)] with two fermions per rung, a dimerized state along the rungs (for $\lambda>t$ ) will be separated from a dimerized state along the bonds $(\lambda<t)$ by a KT phase transition at $\lambda=t$. If in the zig-zag geometry of Fig. 1(b) we place two fermions per well, both in $p$ orbitals, then for $\lambda \ll J_{H}$ due to the Hund's coupling a total $S=1$ state will be formed in each well, and the Haldane phase of a spin-1 chain will be realized.

In conclusion, dipolar fermions on zig-zag lattices can capture relevant spin-orbital models of realistic $d$ electron systems and allow to explore parameter regimes which are hardly accessible for solid-state compounds. Moreover, the quantum nature of orbital fluctuations can be revived, which combined with geometric frustration and spin dynamics produces an intriguing rich ground state phase diagram.

We thank A. Cojuhovschi, G. Khaliullin, A. Kolezhuk, and S. Ospelkaus for useful discussions, and S. Furukawa for correspondence on the Haldane-Dimer state. This work has been supported by QUEST (Center for Quantum Engineering and Space-Time Research). Support from GNSF/ST09-447 (G.J.) and SCOPES Grant IZ73Z0-128058 (T.V.) is acknowledged.

* Also at Andronikashvili Institute of Physics, 0177 Tbilisi, Georgia.

[1] Y. Tokura and N. Nagaosa, Science 288, 462 (2000).

[2] E. Dagotto, Science 309, 257 (2005).

[3] L.F. Feiner, A.M. Oleś, and J. Zaanen, Phys. Rev. Lett. 78, 2799 (1997).

[4] G. Khaliullin and S. Maekawa, Phys. Rev. Lett. 85, 3950 (2000).

[5] F. Wang and A. Vishwanath, Phys. Rev. B 80, 064413 (2009).

[6] P. Corboz, A.M. Läuchli, K. Penc, M. Troyer, and F. Mila, Phys. Rev. Lett. 107, 215301 (2011).

[7] J. Chaloupka, G. Jackeli, and G. Khaliullin, Phys. Rev. Lett. 105, 027204 (2010).

[8] G. Jackeli and D.A. Ivanov, Phys. Rev. B 76, 132407 (2007).

[9] G. Jackeli and D.I. Khomskii, Phys. Rev. Lett. 100, 147203 (2008).

[10] C. Wu, D. Bergman, L. Balents, and S. Das Sarma, Phys. Rev. Lett. 99, 070401 (2007).

[11] M. Hermele, V. Gurarie, and A.M. Rey, Phys. Rev. Lett. 103, 135301 (2009).

[12] A.V. Gorshkov et al., Nature Physics 6, 289 (2010).

[13] C. Chin et al., Rev. Mod. Phys. 82, 1225 (2010).

[14] J. Struck et al., Science 333, 996 (2011).

[15] G.-B. Jo et al., arXiv:1109.1591 (2011).

[16] G. Wirth, M. Ölschläger and A. Hemmerich, Nature
Physics 7, 147 (2010).

[17] T. Lahaye et al., Nature 448, 672 (2007).

[18] M. Lu et al., Phys. Rev. Lett. 107, 190401 (2011).

[19] K.-K. Ni et al., Nature 464, 1324 (2010).

[20] K. Góral, L. Santos, and M. Lewenstein, Phys. Rev. Lett. 88, 170406 (2002).

[21] L. He and W. Hofstetter, Phys. Rev. A 83, 053629 (2011).

[22] M.J. Konstantinović et al., Phys. Rev. B 69, 020409(R) (2004);

[23] T. Hikihara and Y. Motome, Phys. Rev. B 70, 214404 (2004).

[24] G. Jackeli and G. Khaliullin, Phys. Rev. Lett. 103, 067205 (2009).

[25] Ladder-like (zig-zag) lattices may be obtained by combining two-well potentials and square (triangular) lattices.

[26] K.I. Kugel and D.I. Khomskii, Sov. Phys. Usp. 25, 231 (1982).

[27] See attached supplemental material for details.

[28] C.K. Majumdar and D. Ghosh, J. Math. Phys. 10, 1388 (1969).

[29] J.M. Kosterlitz and D.J. Thouless, J. Phys. C 6, 1181 (1973).

[30] S.R. White and I. Affleck, Phys. Rev. B 54, 9862 (1996).

[31] A.A. Nersesyan, A.O. Gogolin, and F.H.L. Eßler, Phys. Rev. Lett. 81, 910 (1998).

[32] S. Furukawa, M. Sato, and S. Onoda Phys. Rev. Lett. 105, 257205 (2010).

[33] The dimerized phase of spin- $\frac{1}{2}$ zig-zag chain for $j_{1}<0$ is dubbed as Haldane-Dimer phase by M. Sato et al., Mod. Phys. Lett. B 25, 901 (2011).

[34] The low-energy fixed point of two decoupled Heisenberg spin- $\frac{1}{2}$ chains belongs to the universality class of a double KT phase transition, with central charge $c=2$.

[35] F. Verstraete, J.J. Garcia-Ripoll, and J.I. Cirac, Phys. Rev. Lett. 93, 207204 (2004).

[36] G. Vidal, Phys. Rev. Lett. 98, 070201 (2007); R. Orús and G. Vidal, Phys. Rev. B 78, 155117 (2008).

[37] Extrapolation follows a $1 / N^{2}$ law, like in the KT transition of $j_{1}-j_{2}$ spin- $\frac{1}{2}$ chain studied by K. Okamoto and K. Nomura, Phys. Lett. A 169, 433 (1992).

[38] W.-L. You, Y.-W. Li, and S.-J. Gu, Phys. Rev. E 76, 022101 (2007).

[39] For $\lambda>1$, before reaching ferro spin state we could not resolve any more dimer order of $(\mathrm{D}, \mathrm{F})$ state. We thus cannot reliably rule out the existence of an additional phase (the ground state though remains a global spin singlet) in a narrow region before reaching the fully polarized spin state $(\mathrm{F}, \mathrm{AF})$ or $(\mathrm{F}$, Para).

[40] As indicated by our iTEBD simulations, for $1<\lambda \lesssim 4$ (around the dashed line of Fig.2(b)), there is an intermediate 4-fold degenerate phase, with a unit cell composed of 4 sites, where apart of the (spontaneous) quadrumerized pattern of nearest neighbour spin correlations, nextnearest spin correlations have a finite dimer order, and orbitals show up-up-down-down ferrimagnetic pattern, with finite $\sum_{i}\left\langle\sigma_{i}^{z}\right\rangle \neq 0$. It however demands considerable numerical resourses to determine accurately the boundaries of this phase, that we leave to future large-scale numerical investigations. 


\section{SUPPLEMENTARY MATERIAL TO "EXPLORING SPIN-ORBITAL MODELS WITH DIPOLAR FERMIONS IN ZIG-ZAG OPTICAL LATTICES"}

In this supplementary material, we provide additional details concerning the derivation of the spin-orbital Hamiltonian and the bosonization procedure.

\section{THE EFFECTIVE SPIN-ORBITAL HAMILTONIANS}

In this section we outline the essential steps of the derivation of the effective spin-orbital Hamiltonian for the two systems, A and B, discussed in the Letter.

\section{A: Snake-like lattice}

We introduce the fermionic annihilation operator $c_{a i, s}$, where $a=1$ (2) indicates the up (down) sites on $i$-th rung, and $s=\uparrow$ or $\downarrow$ refer to the spin. Using the notation of the Letter, system A is described by the Hubbard like Hamiltonian $H=H_{k i n}+H_{\text {int }}$, where

$$
\begin{aligned}
H_{k i n} & =-\lambda \sum_{i, s}\left(c_{1 i, s}^{\dagger} c_{2 i, s}+c_{2 i, s}^{\dagger} c_{1 i, s}\right) \\
& -\frac{t}{2} \sum_{i, a, s}\left[1+(-1)^{i+a}\right]\left[c_{a i, s}^{\dagger} c_{a i+1, s}+c_{a i+1, s}^{\dagger} c_{a i, s}\right]
\end{aligned}
$$

accounts for single-particle processes, and

$$
\begin{aligned}
H_{i n t} & =U \sum_{i, a} c_{a i, \uparrow}^{\dagger} c_{a i, \uparrow} c_{a i, \downarrow}^{\dagger} c_{a i, \downarrow} \\
& +V \sum_{i, s, s^{\prime}} c_{1 i, s}^{\dagger} c_{1 i, s} c_{2 i, s^{\prime}}^{\dagger} c_{2 i, s^{\prime}}
\end{aligned}
$$

corresponds to two-particle interactions. In the limit $U, V \gg t$, and retaining one particle per ladder rung (quarter filling), the effective Hamiltonian up to the second order in $t$ acquires the form of Eq. (1) of the Letter $($ at $\Delta=0)$, where

$$
\begin{aligned}
S_{i}^{z} & =\sum_{a} S_{a i}^{z}=\frac{1}{2} \sum_{a}\left(c_{a i, \uparrow}^{\dagger} c_{a i, \uparrow}-c_{a i \downarrow}^{\dagger} c_{a i, \downarrow}\right) \\
S_{i}^{+} & =\sum_{a} S_{a i}^{+}=\sum_{a} c_{a i, \uparrow}^{\dagger} c_{a i, \downarrow},
\end{aligned}
$$

are the spin- $\frac{1}{2}$ operators on the $i$-th rung, and

$$
\begin{aligned}
\sigma_{i}^{x} & =\sum_{s}\left(c_{1 i, s}^{\dagger} c_{2 i, s}+c_{2 i, s}^{\dagger} c_{1 i, s}\right) \\
\sigma_{i}^{z} & =\sum_{s}\left(c_{1 i, s}^{\dagger} c_{1 i, s}-c_{2 i, s}^{\dagger} c_{2 i, s}\right)
\end{aligned}
$$

are the Pauli matrices corresponding to the pseudoorbital degrees of freedom.

\section{B: $p$-band zig-zag lattice}

In the system $\mathrm{B}$, the pseudo orbital index $a=1$ (2) refers to $p_{x}\left(p_{y}\right)$ orbital in a given well. The kinetic part of the Hamiltonian remains the same as in Eq. (3), while the interaction part, in addition to the terms shown in Eq. (4), acquires the following supplementary terms:

$$
\begin{aligned}
H_{i n t}^{\prime} & =-2 J_{H} \sum_{i}\left[\mathbf{S}_{1 i} \mathbf{S}_{2 i}+\frac{n_{1 i} n_{2 i}}{4}\right] \\
& +J_{\mathrm{H}} \sum_{i}\left[c_{1 i \uparrow}^{\dagger} c_{1 i \downarrow}^{\dagger} c_{2 i \downarrow} c_{2 i \uparrow}+c_{2 i \uparrow}^{\dagger} c_{2 i \downarrow}^{\dagger} c_{1 i \downarrow} c_{1 i \uparrow}\right],
\end{aligned}
$$

where $\mathbf{S}_{1(2) i}$ and $n_{1(2) i}$ are, respectively, spin and density operators for a fermion in $p_{x}\left(p_{y}\right)$ orbital state. The first line stands for Hund's exchange and the second one describes the so-called pair-hopping [1].

The coupling constants $U, V$, and $J_{H}$, entering in Eqs. (45), can be expressed in terms of a two-body potential and single-particle wave functions [1]. One finds:

$$
\begin{aligned}
& U=\int \mathrm{d} \mathbf{r}_{\mathbf{1}} \mathrm{d} \mathbf{r}_{\mathbf{2}} P_{x(y)}^{2}\left(\mathbf{r}_{\mathbf{1}}\right) V\left(\mathbf{r}_{\mathbf{1}}-\mathbf{r}_{\mathbf{2}}\right) P_{x(y)}^{2}\left(\mathbf{r}_{\mathbf{2}}\right), \\
& V=\int \mathrm{d} \mathbf{r}_{\mathbf{1}} \mathrm{d} \mathbf{r}_{\mathbf{2}} P_{x}^{2}\left(\mathbf{r}_{\mathbf{1}}\right) V\left(\mathbf{r}_{\mathbf{1}}-\mathbf{r}_{\mathbf{2}}\right) P_{y}^{2}\left(\mathbf{r}_{\mathbf{2}}\right),
\end{aligned}
$$

and

$$
J_{H}=\int \mathrm{d} \mathbf{r}_{1} \mathrm{~d} \mathbf{r}_{2} P_{x}\left(\mathbf{r}_{1}\right) P_{y}\left(\mathbf{r}_{1}\right) V\left(\mathbf{r}_{1}-\mathbf{r}_{2}\right) P_{x}\left(\mathbf{r}_{\mathbf{2}}\right) P_{y}\left(\mathbf{r}_{\mathbf{2}}\right) .
$$

Above $P_{x}$ and $P_{y}$ are orbital wavefunctions of the same well, and $V\left(\mathbf{r}_{1}-\mathbf{r}_{\mathbf{2}}\right)$ is the total interparticle potential (including both contact as well as dipolar interactions).

The energy of the state with two fermions occupying the same orbital is $U$. However, the pair-hopping term moves two particles from the doubly occupied orbital to an empty orbital with the amplitude $J_{H}$, and true eigenstates become orbital-symmetric and orbitalantisymmetric states with corresponding energies $U+J_{H}$ and $U-J_{H}$.

Two fermions occupying different orbitals may form a spin-singlet or a spin-triplet state with corresponding energies $V+J_{H}$ and $V-J_{H}$ [2].

In the limit $U \pm J_{H}, V \pm J_{H} \gg t$, and up to second order in $t$, the effective Hamiltonian acquires the form $H=\sum_{i} H_{i, i+1}$, where $H_{i, i+1}$ is the two-site Hamiltonian:

$$
\begin{aligned}
H_{i, i+1} & =-\frac{t^{2}}{\tilde{U}} P_{i, i+1}\left(S^{T}=0\right)\left(1+(-1)^{i} \sigma_{i}^{z}\right)\left(1+(-1)^{i} \sigma_{i+1}^{z}\right) \\
& -\frac{t^{2}}{2\left(V+J_{H}\right)} P_{i, i+1}\left(S^{T}=0\right)\left(1-\sigma_{i}^{z} \sigma_{i+1}^{z}\right) \\
& -\frac{t^{2}}{2\left(V-J_{H}\right)} P_{i, i+1}\left(S^{T}=1\right)\left(1-\sigma_{i}^{z} \sigma_{i+1}^{z}\right) \\
& -\frac{\lambda}{2}\left(\sigma_{i}^{x}+\sigma_{i+1}^{x}\right),
\end{aligned}
$$




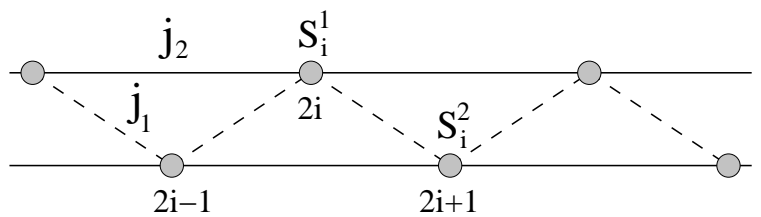

FIG. 3: Two sub-chains with intrachain coupling $j_{2}$ (bold lines) coupled by zig-zag interchain coupling $j_{1}$ (dashed line).

where we have introduced the operators $P_{i, i+1}\left(S^{T}=0\right)=$ $-\mathbf{S}_{i} \mathbf{S}_{i+1}+1 / 4$, and $P_{i, i+1}\left(S^{T}=1\right)=\mathbf{S}_{i} \mathbf{S}_{i+1}+3 / 4$, which project onto two-fermion states on sites $i$ and $i+1$ with, respectively, total spin $S^{T}=0$ and $S^{T}=1$, and $\tilde{U}=$ $\left(U^{2}-J_{H}^{2}\right) / U$.

The first line in Eq. (9) accounts for the situation in which both fermions occupy same orbitals on sites $i$ and $i+1$. In that case, when a fermion hops to the neighboring site [3], the orbital-symmetric or orbitalantisymmetric states of the spin-singlet pair is reached (note that the formation of the spin-triplet pair is forbidden by Pauli exclusion).

The second and third lines of Eq. (9) account for the configuration in which two fermions occupy orthogonal orbitals on neighboring sites. When a fermion hops to a (singly) occupied neighboring site (remembering that inter-site hopping $t$ does not change orbital quantum number), depending on the total spin of the two fermions the interaction may take place either in the spin-singlet channel, with energy cost $V+J_{H}$, or in the spin-triplet channel, with energy cost $V-J_{H}$. A proper re-ordering of the terms in Eq. (9) leads to the Hamiltonian of Eq. (1) of the Letter.

\section{BOSONIZATION ANALYSIS}

In the following we provide some details on our bosonization analysis of the large $\lambda$ regime $(\lambda \rightarrow \infty)$, and in particular on how this approach provides an understanding of the special fine-tuning line $\Delta=\Delta^{*}$ cutting the $(\mathrm{D}, \mathrm{F})$ phase at which spin dimerization and orbital ferro orders vanish.

Integrating out the orbitals in large $\lambda$ limit, to the second order in $1 / \lambda$ we obtain the effective spin- $\frac{1}{2}$ model given in Eq. (2) of the Letter with,

$$
\begin{aligned}
& j_{1}=2(1-\Delta)+\frac{4+\Delta(1+\Delta)}{2 \lambda}+O\left(\lambda^{-2}\right), \\
& j_{2}=\frac{1}{\lambda}+O\left(\lambda^{-2}\right), \quad j_{3}=\frac{1+\Delta}{2 \lambda^{2}}+O\left(\lambda^{-3}\right), \\
& \Omega=\frac{2}{\lambda^{2}}+O\left(\lambda^{-3}\right),
\end{aligned}
$$

where we use the same notations as in the Letter. $\lambda \rightarrow \infty$ is a convenient limit for bosonization, in particular for $j_{1} \ll j_{2}$. In that case, one can consider two weakly coupled chains (see Fig. 3), which we denote as $\mathbf{S}_{i}^{1}=\mathbf{S}_{2 i}$ and
$\mathbf{S}_{i}^{2}=\mathbf{S}_{2 i+1}$. Using bosonization identification, the spins along each chain are represented by smooth and staggered parts $\mathbf{S}_{i}^{a} \rightarrow \mathbf{J}_{L}^{a}+\mathbf{J}_{R}^{a}+(-1)^{i} \mathbf{n}^{a}[4]$. The coupling of the two chains induces marginal operators. Apart from the scalar (dimerization) operators, there are spinfull marginal terms, so-called twist operators [5]. Up to irrelevant and marginal couplings that induce only velocity renormalization, the effective bosonized Hamiltonian density describing the spin degrees of freedom in the large- $\lambda$ limit is of the form

$$
\begin{aligned}
& \mathcal{H}=\frac{\pi v}{2} \mathbf{J}_{\xi}^{a} \mathbf{J}_{\xi}^{a}+D_{1}\left(\mathbf{J}_{L}^{1} \mathbf{J}_{R}^{2}+\mathbf{J}_{R}^{1} \mathbf{J}_{L}^{2}\right)+D_{2} \mathbf{J}_{L}^{a} \mathbf{J}_{R}^{a} \\
& +T_{1} \varepsilon_{a b}\left(\epsilon^{a} \partial_{x} \epsilon^{b}+\boldsymbol{n}^{a} \partial_{x} \boldsymbol{n}^{b}\right)+T_{2} \varepsilon_{a b}\left(3 \epsilon^{a} \partial_{x} \epsilon^{b}-\boldsymbol{n}^{a} \partial_{x} \boldsymbol{n}^{b}\right),
\end{aligned}
$$

where $v \sim j_{2}$, a summation convention is assumed for repeated indices $a, b=(1,2)$, and $\xi=(L, R), \varepsilon_{a b}$ is the antisymmetric symbol, and $\epsilon^{a}$ stand for the dimerization operators, such that $\mathbf{S}_{i}^{a} \mathbf{S}_{i+1}^{a} \sim(-1)^{i} \epsilon^{a}(x)+$ a less relevant smooth part.

Bare values of the twist couplings $T_{1,2}$, and the interchain dimerization $D_{1}$ depend on $j_{1}, j_{3}$ and $\Omega$, in linear order (with non-universal proportionality coefficients), whereas the intra-chain dimerization amplitude $D_{2}$ depends on $j_{2}$ and $\Omega$.

One-loop RG equations corresponding to the effective model (11) are identical to those obtained in [5] for the $S U(2)$ symmetric case. RG flow is dominated by interchain dimerization $D_{1}$, which in the infrared limit always scales to strong coupling, except for the initial condition $T_{1}=T_{2}$, corresponding to the fine tuning $\Delta=\Delta^{*}$, which we interpret as an infrared decoupling point of the two chains. In contrast $D_{2}$ scales to zero at low energies for $\lambda \rightarrow \infty$. Thus for $\lambda \rightarrow \infty$ there is a special line in $(\mathrm{D}, \mathrm{F})$ phase where spin dimerization (and consequently the ferro orbital order) vanishes.

In fact, bosonization is only needed to capture the influence of $j_{3}$ and $\Omega$ terms close to $j_{1}=0$. Away of that region, for $\lambda \rightarrow \infty$, one just needs to retain $j_{1}$ and $j_{2}$ terms, and borrow known results from the frustrated $j_{1}-j_{2}$ spin- $\frac{1}{2}$ chain. This gives us an estimate of other phase transition lines (we use the notations of the Letter) $\Delta_{K T}$ and $\Delta_{F}$ which follow respectively from $j_{1} \simeq 4 j_{2}$ and $j_{1}=-4 j_{2}$ conditions. Whereas $\Delta_{M G}$ (which does not represent a phase transition line) follows from the condition $j_{1}=2 j_{2}$.

* Also at Andronikashvili Institute of Physics, 0177 Tbilisi, Georgia.

[1] See, e.g., C. Castellani, C. R. Natoli, and J. Ranninger, Phys. Rev. B 18, 4945 (1978).

[2] For the spin-triplet state, the coordinate wave-function must have a node when the positions of the two fermions coincide. Hence, for the spin-triplet state the contact $s$ wave interaction does not play any role. This is reflected 
in Eqs. (77) and (8) where for $V\left(\mathbf{r}_{1}-\mathbf{r}_{2}\right) \sim \delta\left(\mathbf{r}_{1}-\mathbf{r}_{2}\right)$, $V=J_{H}$ and hence scattering in the spin-triplet channel vanishes.

[3] Note that hopping from a site $i$ to a site $i+1$ is only possible if the fermion occupies on site $i$ the orbital that is directed towards the site $i+1$, as depicted on Fig. 1(b) of the Letter.
[4] A.O. Gogolin, A.A. Nersesyan, and A.M. Tsvelik, Bosonization and Strongly Correlated Systems, Cambridge University Press (1998); T. Giamarchi, Quantum Physics in One Dimension, Oxford University Press (2003).

[5] A.A. Nersesyan, A.O. Gogolin, and F.H.L. Eßler, Phys. Rev. Lett. 81, 910 (1998). 